\title{
A design method for thin high-order optical film filters under boundary conditions
}

\author{
B. Diehm ${ }^{1 *}$, C. Gulde, and N. Frühauf ${ }^{1}$ \\ ${ }^{I}$ Chair of Display Technology and Research Center SCoPE, Universität Stuttgart, Allmandring 3b, 70569 Stuttgart
}

Received May 23, 2011; accepted June 22, 2011; published June 30, 2011

\begin{abstract}
This paper presents an efficient search method for equivalent thin film filter realization to a given design under boundary conditions. To calculate realizations, the algorithm presented in [1] is used and the boundary conditions are given in the form of refractive index intervals. Since the number of possible solutions increases exponentially with the number of film layers, only a small part of them can be checked for boundary conditions in a reasonable time. The method presented here uses the results of a Monte Carlo simulation and Yen's algorithm. The time consuming simulation is conducted only once for given boundary conditions. Since the boundary conditions depend on the fabrication process this method saves a significant amount of time during filter design.
\end{abstract}

In [1] an exact synthesis algorithm for dielectric thin film filters with a uniform optical phase thickness is presented, based on a synthesis method for lossless electric lines [3] and reactance networks [4]. This algorithm has the benefit of providing all possible solutions in terms of thin film filter stacks to a given transmission or reflection characteristic under the restrictions stated above. In [1] it is only assured that the refractive indices of all solutions have strictly positive values. Unfortunately, this is insufficient for technological realization since the refractive indices of practical materials only exist as discrete values. One way to alleviate this restriction is to use a mixture of materials with different refractive indices for layer fabrication [5-7]. Another way is to replace a given refractive index layer by a symmetric system of three layers by application of the Herpin equivalent index theorem [8-11]. Both ways are especially suited to creating arbitrary refractive index values in a certain interval which is bounded by two refractive indices $n_{\mathrm{L}}$ and $n_{\mathrm{H}}$. From a technological point of view this subset of realizable solutions is of substantial interest. Instead of refractive indices $n$ optical admittances $\eta$ known from [12], which are given by $\eta=n \cdot \sqrt{\varepsilon_{0} \cdot \mu_{0}^{-1}}$ will be used in the following. The boundary condition in this paper is therefore given by Eq. (1).

$$
0<\eta_{\mathrm{L}} \leq \eta_{j} \leq \eta_{\mathrm{H}^{\prime}} j=1, \ldots, N
$$

The synthesis algorithm presented in [1] states necessary and sufficient conditions for the feasibility of the filter transmission $T$ as a function of the uniform phase

\footnotetext{
*E-mail: 1fb@1fb.uni-stuttgart.de; http://www.lfb.uni-stuttgart.de
}

thickness $\delta$ which is given in Eq. (2).

$$
\delta=2 \pi \cdot n \cdot d \cdot \lambda^{-1}
$$

As mentioned above, only strictly positive admittance values are guaranteed. With respect to Eq. (1) the following questions arise. Are there any thin film stacks among the equivalent solutions which fulfill Eq. (1)? Is there an efficient search method for these solutions, especially if the filter order with respect to the number of thin film layers $N$ is high? Regarding the first question, only precise necessary conditions can be stated for low order filters by using various algebraic and numeric methods at the moment. This subject will be treated in a subsequent paper.

First consider the derivation of the characteristic matrix $\boldsymbol{M}$ and the transmission $T$ for a general dielectric thin film filter stack with $N$ layers presented in [12]. The stack is embedded in an ambient medium with the admittance $\eta_{0}$ on one side and a substrate medium with $\eta_{N+1}$ on the other side. By demanding uniform optical phase thickness for all layers, the frequency variable $\delta$ as given in Eq. (2) can be used. Now a well known frequency transformation $s=\sigma+\mathrm{i} \Omega=\tan (\mathrm{i} \delta)$ presented in [3] and [13] with $s$ as a new complex frequency variable and $\Omega=\operatorname{Im}\{s\}$ is introduced. By mapping the interval $\delta=[-\pi / 2 ; \pi / 2]$ onto the whole imaginary axis in the $s$-plane, this frequency transformation links the optical synthesis problem considered here to well known concepts for the synthesis of lossless transmission lines with uniform time delay and reactance filters [4], [13]. By using this approach, we get $\boldsymbol{M}(s), \tau(\delta)$ and $T(\delta)$ as given in Eqs. (3), (4) and (5).

$$
\begin{gathered}
\mathbf{M}(s)=\frac{1}{{\sqrt{1-s^{2}}}^{N}} \cdot \prod_{r=1}^{N}\left(\begin{array}{cc}
1 & s \cdot \eta_{r}^{-1} \\
s \cdot \eta_{r} & 1
\end{array}\right)=\left(M_{i j}\right) \\
\tau(\delta)=\left[\frac{2{\sqrt{1-s^{2}}}^{N}}{M_{11}+\eta_{N+1} M_{12}+\frac{1}{\eta_{0}} M_{21}+\frac{\eta_{N+1}}{\eta_{0}} M_{22}}\right]_{s=\tan (\mathrm{i} \delta)} \\
T(\delta)=\frac{\eta_{N+1}}{\eta_{0}} \cdot \tau(\delta) \cdot \tau^{*}(\delta)
\end{gathered}
$$

For the filter synthesis procedure, the algorithm presented in [1] states necessary and sufficient conditions for the 
filter's transmission function so we start with a given transmission function $T(\delta)$. From there, the so called response function $K(s)$ of the filter is derived in [1] in a unique way. The response function is related to the transmission coefficient by Eq. (6) and it is the initial point for derivation of a set of equivalent solutions.

$$
\tau(\delta)=\left[\frac{1}{K(s)}\right]_{s=\tan (\mathrm{i} \delta)}
$$

First, a so-called characteristic function $f(s)$ is introduced, which is related to the response function by equation (7).

$$
f(s) \cdot f(-s)=K(s) \cdot K(-s)-\eta_{0}^{-1} \eta_{N+1}
$$

From Eqs. (3), (4) and (6) it follows that the numerator of Eq. (7) is a polynomial in $s$. By assigning the roots of the numerator polynomial on the right side in Eq. (7) to $f(s)$ and $f(-s)$ on the left side, a particular equivalent filter solution is selected. Finally, by considering the response function and one particular characteristic function a unique filter realization can be derived with the algorithms presented in [1]. There it is also pointed out that the constant numerator coefficients of Eq. (7) are fixed, so $K(s)$ and $f(s)$ are fully characterized by the roots of their numerator polynomials. These roots and their distribution on the real axis and in the complex plane will be considered in the remaining part of the paper.

The next step is to get a prediction if there can exist at least one solution which fulfills Eq. (1), and how to efficiently search for characteristic functions which fulfill Eq. (1) in the equivalent set of all solutions. This is done by conducting a Monte Carlo simulation for the roots of $K(s)$ and $f(s)$ with given boundary conditions according to (1) for a fixed number of $N$ layers.

In a Monte Carlo simulation the root probability distributions of the numerator polynomials of $K(s)$ and $f(s)$ for realizable thin film layer stacks according to Eq. (1) are approximated. The first step in every experiment in the simulation is to generate optical admittances of a realizable thin film layer stack by using random numbers. Random numbers are independent and uniformly distributed in the interval given by Eq. (1). For such a filter stack the numerator polynomials $K_{\text {num }}(s)$ and $f_{\text {num }}(s)$ can be calculated by using the Eq. (8) and (9), which can be derived from [1].

$$
\begin{aligned}
& K_{\text {num }}(s)=\frac{1}{2}\left[M_{11}(s)+\eta_{N+1} M_{12}(s)+\frac{1}{\eta_{0}} M_{21}(s)+\frac{\eta_{N+1}}{\eta_{0}} M_{22}(s)\right] \\
& f_{\text {num }}(s)=\frac{1}{2}\left[M_{11}(s)+\eta_{N+1} M_{12}(s)-\frac{1}{\eta_{0}} M_{21}(s)-\frac{\eta_{N+1}}{\eta_{0}} M_{22}(s)\right]
\end{aligned}
$$

Next the roots of Eqs. (8) and (9) are calculated and separated into roots on the real axis and in the complex $s$ plane without the real axis. The steps previously described are repeated $S$ times in the simulation and the roots are collected. These four generated sets of roots are now used to create two histograms each for $K_{\text {num }}(s)$ and $f_{\text {num }}(s)$. The histograms for the real axis have dimension one while the ones for the complex plane have dimension two. To get an accurate description of the shape of the root probability distributions a dynamic binning method can be used. If the number of roots in one bin exceeds a specified maximum value, the bin is subdivided into a number of smaller bins of equal size. This procedure is repeated until a preset minimum bin size has been reached. In this way the areas of distribution with a high number of roots are described in greater detail than the areas with only a few roots. By dividing the number of roots in one bin by its length resp. area, we get the root density for each bin. Since the steps described in this section to generate the histograms depend only on the number of thin film layers $N$ and the boundary conditions stated in Eq. (1), the Monte Carlo simulation has to be done only once.

Consider a filter response function $K_{0}(s)$ which fulfills the necessary and sufficient conditions stated in Ref. [1]. This function can be derived, for example, from a given transmission characteristic which has now to be realized as a thin film filter. By calculating the roots of $K_{0}(s)$ and searching the associated bins in the generated histograms, we can get a prediction about the overall realizability of the filter function. If some of the roots of $K_{0}(s)$ are associated with bins of the histogram with a very low or even zero root density, the chance is high that there exists no equivalent solution which is at all realizable according to Eq. (1). If the overall realizability for the considered $K_{0}(s)$ is high enough, we can start the search for equivalent solutions.

To efficiently search for equivalent solutions with a high chance of realizability according to Eq. (1), all possible roots of the characteristic function $K_{0}(s)$ are calculated by using Eq. (7). Again, to every root $s_{0}$ of Eq. (7), the associated bin with its root density $\rho\left(s_{0}\right)$ in the one- and two-dimensional histogram is determined. Different equivalent solutions are only created by roots $s_{0}$ of $f(s)$ which are not located on the imaginary axis because for roots on the imaginary axis there is no difference between assigning them to $f(s)$ or $f(-s)$. Now assume that one of these roots $s_{0}=\sigma_{0}+\mathrm{i} \Omega_{0}$ lies in the right open half plane with an associated bin in the histogram with the root density $\rho\left(\sigma_{0}+\mathrm{i} \Omega_{0}\right)$. The corresponding root in the left open half plane, therefore, is located at $s=-\sigma_{0}+\mathrm{i} \Omega_{0}$ with the root density $\rho\left(-\sigma_{0}+\mathrm{i} \Omega_{0}\right)$. Now for every selection between a root in the right open half plane and the corresponding root in the left open half plane, a probability of feasibility $P\left(s_{0}\right)$ is stated by Eq. (10).

$$
P\left(s_{0}\right)=\frac{\rho\left(\sigma_{0}, \Omega_{0}\right)}{\rho\left(\sigma_{0}, \Omega_{0}\right)+\rho\left(-\sigma_{0}, \Omega_{0}\right)}
$$


In the case of $\rho\left(\sigma_{0}, \Omega_{0}\right)=\rho\left(-\sigma_{0}, \Omega_{0}\right)=0$, the probability value of $P\left(s_{0}\right)=1 / 2$ is assigned to the corresponding selection. Note that $f_{\text {num }}(s)$ has to be a polynomial with real coefficients in $s$ so it is sufficient to consider only one root of every complex conjugated root pair. By multiplying all possible root selections an overall probability of feasibility for every equivalent solution can be stated by Eq. (11). The set $E$ contains a decision for every root selection, which is necessary to determine $f_{\text {num }}(s)$ in a unique way. That is to say, if the root in the right or the left open half plane was selected for $f_{\text {num }}(s)$.

$$
P_{E}=\prod_{s_{0 i} \in E} P\left(s_{0 \mathrm{i}}\right)
$$

Since for every set $E$ a probability $P_{E}$ can be calculated and every set $E$ links to one equivalent solution, all equivalent solutions can be ordered according their probability of realizability derived by the histograms of the Monte Carlo simulation. Now we have to find the $K$ selection sets with the highest probability $P_{E I} \geq \ldots \geq P_{E K}$ of realizability. This problem can be described by using a binary decision graph as pictured in Fig. 1.

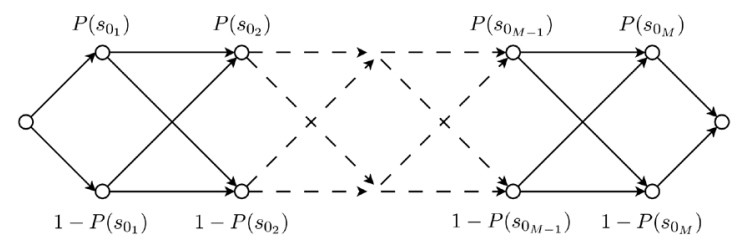

Fig. 1. The problem of finding the $K$ root selection sets with the highest probability of feasibility is transformed into a binary graph.

Because single root selections are binary decisions (for the left or the right open half plane) and are considered independent of each other, the graph has a simple structure with interchangeable segments. The $K$ shortest paths can be found with a simplified version of Yen's algorithm. Yen's algorithm finds the $K$ shortest loopless paths between two nodes in a graph. As a result, we get the $K$ decision sets and, further, the related characteristic functions with the highest probability of feasibility. Now we can derive a thin film layer stack from every equivalent solution found. Since the method presented here only gives a probability of feasibility, we have to check every solution if it complies with the given boundary conditions.

The results presented here concern only the problem of finding feasible solutions according to Eq. (1). To ensure that there exists, at least, one feasible solution in the solution set, at first a thin film stack with $N=30$ layers was generated. The optical admittances of all layers were randomly chosen from the interval [1.4;2.6]. The admittances of the ambient and substrate media were selected to air with $\eta_{0}=1.00$ and glass with $\eta_{N+1}=1.52$. Now the associated response function $K_{0}(s)$ for this filter stack was calculated and used as a starting point like in the previous section. To generate sufficient data in the Monte Carlo Simulation $S \approx 10^{4}$ experiments were created from i.i.d. random variables which were uniformly distributed in the interval $[1.7 ; 2.3]$. Again, $\eta_{0}$ and $\eta_{N+1}$ were selected as above. Now the search method presented in the previous section was used to find feasible solutions. A solution was considered feasible if Eq. (1) with $\eta_{\mathrm{L}}=1.4$ and $\eta_{\mathrm{H}}=2.6$ was met for all layers. To compare the results, also a standard linear search was conducted. The linear search runs through all possible root selections without using the information provided by the histograms (see Fig. 2).

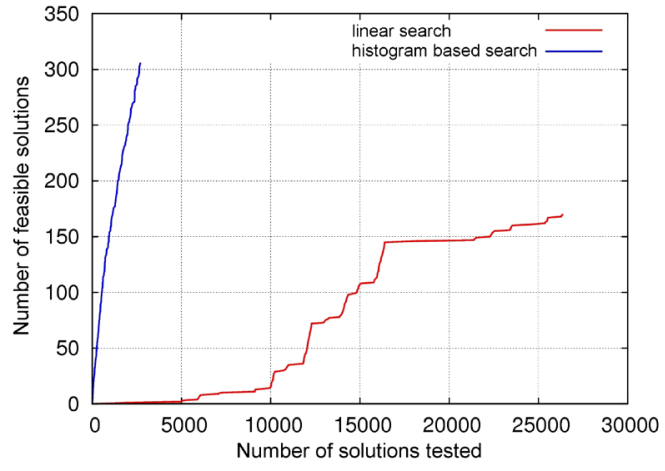

Fig. 2: Comparison between the histogram based search method and a standard linear search.

The number of examined solutions is plotted against the number of feasible solutions found. Both search methods were stopped after ten minutes runtime. As depicted in Fig. 2, the histogram based search finds about twice as much feasible solutions in the same time. In fact, to retrieve the next solution, the histogram based search needs more time than the linear search but this is outweighed by the higher probability of feasibility. All the simulations were implemented in Octave. By using dynamic binning, more sophisticated generation of histograms, search algorithms and $\mathrm{C}++$ language, the performance can be farther improved. Since the boundary conditions only change if the technological setup has to be changed, the time consuming Monte Carlo simulation has to be conducted only in this case.

\section{References}

[1] A. Rowinska-Schwarzweller, Proc. SPIE 12 (2004).

[2] J.Y.Yen, Mangement Science 17, 712 (1971).

[3] P.I. Richards, Proc. of the IRE 36(2), 217 (1948).

[4] W. Bader, Archiv für Elektrotechnik 34(4), 181 (1940).

[5] V. Yadava, S. Sharma, K. Chopra, Thin Solid Films 17, 243 (1973).

[6] S. Lange et al., Thin Solid Films 502, 29 (2006).

[7] H. Terui, M. Kobayashi, App. Phys. Lett. 32, 666 (1978).

[8] A. Herpin, Comptes Rendus 225, 182 (1947).

[9] J.A. Dobrowolski, S.H.C. Piotrowski, App. Opt. 21, 1502 (1982).

[10] L.I. Epstein, J. Opt. Soc. Am. 42, 806 (1952).

[11] M.C. Ohmer, J. Opt. Soc. Am. 68, 137 (1978)

[12] H.A. Macleod, Thin Film Optical Filters (Institute of Physics Publishing, 2001).

[13] R. Unbehauen, Netzwerk und Filtersynthese (R. Oldenburg, Verlag, Wien 1993). 